\title{
Effect of Hydrokinesitherapy on the Rehabilitation Outcome in Patients After Total Hip Replacement
}

\author{
Dragana Dragičević-Cvjetković, ${ }^{1,2}$ Slavko Manojlović, ${ }^{2}$ Monika Gligić ${ }^{1}$
}

\begin{abstract}
Background/Aim: Total hip replacement is considered as one of the most successful treatment methods in orthopaedic surgery of the 20th century, since it significantly improves the quality of life of the individuals with coxarthrosis. Protocols and the organisation of the postoperative rehabilitation differ significantly around the world. The aim of this study was to investigate the effect of hydrokinesitherapy on the rehabilitation outcome in patients after total hip replacement under in-patient conditions.

Methods: This prospective clinical trial included a total of 100 patients of both genders, average age $59.11 \pm 8.85$ years, which were admitted to the in-patient postoperative rehabilitation after total hip replacement. Patients were randomised in two groups: group A $(n=50)$ that was subjected to rehabilitation program with hydrokinesiherapy and group $B(n=50)$ that was subjected to rehabilitation program only. The parameters registered were: the range of movement in the operated hip, the circumference of the femoral musculature and the Western Ontario and McMaster Universities Osteoarthritis Indeks (WOMAC index) at admittance and discharge from in-patient rehabilitation.

Results: The average values of hip flexion with extended knee, extension and external hip rotation were significantly better in group A $(p<0.05)$. The values of the circumference of the femoral musculature and WOMAC index in patients from group A were significantly better on discharge compared to the admission. Also, this group had a statistically significantly better values of the circumference of the femoral musculature and value of WOMAC index at discharge compared to patients from group B $(\mathrm{p}<0.05)$.

Conclusion: Hydrokinesitherapy as a part of the protocol for postoperative rehabilitation in patients after total hip replacement leads to a faster improvement of the functional status of the operated hip and patient's quality of life.
\end{abstract}

Key words: Rehabilitation; Hydrotherapy; Replacement; Hip.
(1) Institute for Physical Medicine and Rehabilitation "Dr Miroslav Zotović" Banja Luka, Republic of Srpska, Bosnia and Herzegovina.

(2) Faculty of Medicine, University of Banja Luka, Banja Luka, Republic of Srpska, Bosnia and Herzegovina.

Correspondence:

DRAGANA

DRAGIČEVIĆ-CVJETKOVIĆ

E: dragicevicdr@gmail.com

M: +387 65711138

\section{ARTICLE INFO}

Received: 7 November 2020

Revision received: 28 November 2020 Accepted: 7 December 2020

\section{Introduction}

Quality of treatment of patients with advanced coxarthrosis is high only in case of the synergistic effect the well-planned and executed orthopaedic treatment and well-organised postoperative rehabilitation. Total hip replacement is considered as one of the most successful orthopaedic procedures today. ${ }^{1}$ The goals of postoperative rehabilitation are decreased pain, improved mobility, muscular strength and quality of life of these patients. Decreased the femoral circumference as a direct index of hypotrophy of the femoral muscles is one of the important parameters of the functional status 
of patients after total hip replacement. Postoperative rehabilitation program includes the use of interferential currents, magnetotherapy, kinesitherapy, hydrokinesitherapy, occupational therapy and other modalities as needed. ${ }^{2}$ Hydrokinesitherapy (aquatic, therapeutic exercises in pool) is one of the therapeutic modalities whose physical characteristics decrease the pressure on the operated hip and enables earlier, more intensive and less painful movements. Besides, selected exercises in the water medium assure normalisation of the patterns of muscular activation, mobility and stability of the hip joint and proprioception. ${ }^{3}$ Aim of the study was to investigate the effect of hydrokinesitherapy on the outcome of the rehabilitation of patients after total hip replacement under the in-patient conditions.

\section{Methods}

This prospective clinical trial included a total of 100 patients of both genders, aged (mean \pm standard deviation) $59.11 \pm 8.85$ years with advanced unilateral coxarthrosis that underwent total, primarily, unilateral hip replacement and were admitted on in-patient postoperative rehabilitation. Inclusion criteria were: normal laboratory parameters (sedimentation of erythrocytes, C-reactive protein, complete blood cell count and urinalysis), normal postoperative radiographic imaging of the operated hip and normal control orthopaedic and physiatric examination. Non-inclusion criteria were: immobile patients, infection, patients with inflammatory rheumatism and patients who could not undergo the in-patient postoperative rehabilitation with hydrokinesitherapy due to comorbidities. The research was approved by the Managing Director of the Institute for Physical Medicine and Rehabilitation "Dr Miroslav Zotovic", Banja Luka.

All the patients were operated at the Orthopaedic Surgery Department and treated at the Department for Treatment of Rheumatologic, Postoperative and Posttraumatic conditions of the Institute for Physical Medicine and Rehabilitation "Dr Miroslav Zotović" in Banja Luka during 2016. Patients were divided by means of the block randomisation in two groups of 50 patients each, depending on the fact whether they were treated with hydrokinesitherapy during the in-patient postoperative rehabilitation or not. The protocol of the in-patient postoperative rehabilitation comprised the implementation of: individually-adjusted ki- nesitherapy, occupational therapy, interferential currents aimed at the operated hip and magnetotherapy in the form of low-frequency magnetic field once daily over a three-week period. ${ }^{4-6} \mathrm{~Pa}-$ tients in group A $(n=50)$ had hydrokinesitherapy once daily during 30 minutes in total period of 21 days, while patients in $B(n=50)$ did not have it. Hydrokinesitherapy included exercises to increase the range of motion and strengthen the muscle strength of pelvic and femoral muscles in water of indifferent temperature $\left(34-35^{\circ} \mathrm{C}\right)$, which patients performed in "Slatina" mineral water under the supervision of a physiotherapist once a day for 30 minutes. According to its composition, this mineral water is a sodium-calcium-magnesium hydrocarbonate hypertherm that is cooled to an indifferent temperature for therapeutic purposes. Monitored parameters of the effect of postoperative rehabilitation were: range of movement in the operated hip, the circumference of the femoral musculature and the Western Ontario and McMaster Universities Osteoarthritis Index (WOMAC index) on admission and discharge from the in-patient rehabilitation. The range of motion and circumference of the femoral musculature were parameters of functional recovery of the operated hip and WOMAC index was used as the standard test of quality of life measurement in these group of patients. The range of motion was measured with a goniometer and the circumference of the femoral musculature with a measuring tape in centrimetres in three consecutive measurements by the same examiner. The values of the monitored parameters were the average values of three consecutive measurements. The circumference of the femoral musculature with a centimetre tape was measured on $10 \mathrm{~cm}$ above top border of the patella. The data were collected on admission and on discharge form in-patients rehabilitation. Statistical data processing was done using the Statistical Package for the Social Sciences (SPSS) programme version 20. Methods of descriptive statistics, Chisquared test, Levene's test, t-test, Mann-Whitney test and Spearman's correlation were used for statistical analysis of the data. As a statistically significant difference was considered $\mathrm{p}<0.05$.

\section{Results}

\section{Demographic characteristics}

Out of a total of 100 patients after primarily, unilateral, total hip replacement, between the two 
equal groups - A and B - there was no significant difference as regards to gender and body mass index (BMI), which speaks in favour of the homogeneity of the groups. Patients in group A were significantly older $(\mathrm{p}<0.05)$. (Table 1$)$.

Table 1: Distribution of the patients according to age, body mass index (BMI) and gender

\begin{tabular}{|c|c|c|c|c|c|}
\hline & \multirow[b]{2}{*}{$\mathbf{N}$} & \multirow{2}{*}{$\begin{array}{c}\text { Age } \\
\text { Mean } \pm \text { SD }\end{array}$} & \multirow{2}{*}{$\begin{array}{c}\text { BMI } \\
\text { Mean } \pm \text { SD }\end{array}$} & \multicolumn{2}{|c|}{ Gender } \\
\hline & & & & Female N (\%) & Male N (\%) \\
\hline Group A & 50 & $62.62 \pm 7.51$ & $27.62 \pm 1.98$ & $26(48.1 \%)$ & $24(52.2 \%)$ \\
\hline Group B & 50 & $55.60 \pm 8.75$ & $28.19 \pm 1.65$ & 28 (51.9\%) & $22(47.8 \%)$ \\
\hline$p$-value & & $0.009^{*}$ & $0.11^{*}$ & \multicolumn{2}{|c|}{$0.84^{\star \star}$} \\
\hline
\end{tabular}

\section{Amplitude of movement in the operated hip}

The values of flexion with extended knee in the operated hip on admission were not significantly different between the patient groups A and B (47.80 \pm 6.93 vs $39 \pm 9.42$ degrees). Patients in both groups had larger amplitude of flexion with extended knee after the implementation of the in-patient rehabilitation, but this improvement was significantly better in Group A patients. Increase of this parameter on discharge was $39.02 \%$ and $18.87 \%$ of the admission values in Groups A and B, respectively (Figure 1).

Mean value of the external rotation in the operated hip was also significantly improved after the implementation of the in-patient rehabilitation in patient exposed to hydrokinesither-

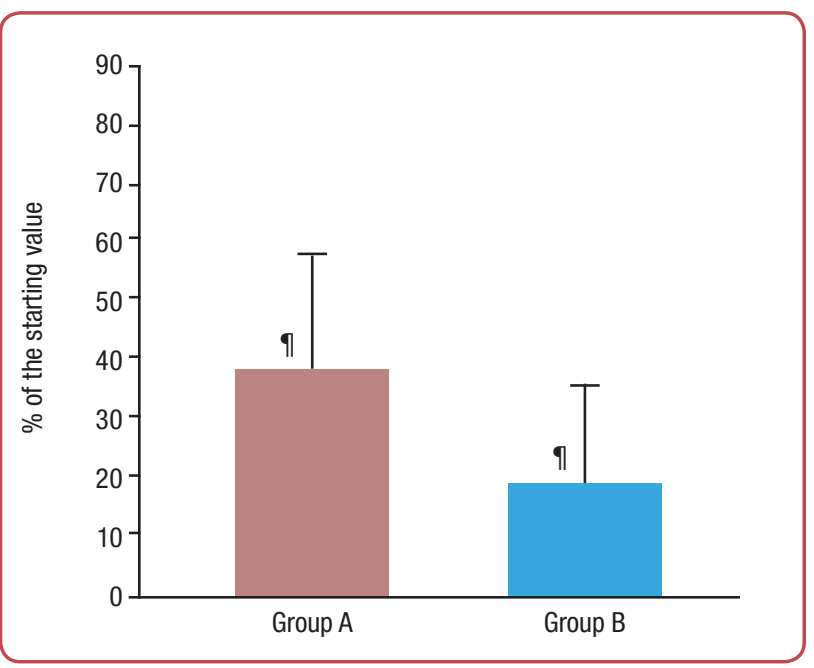

Figure 1: Increase in the amplitudes of the operated hip flexion (\% of values before rehabilitation) with extended knee in patients with hip replacement after in-patient rehabilitation. Group A - rehabilitation programme plus hydrokinesitherapy, Group B - rehabilitation programme without hydrokinesitherapy. $9 p<$ 0.05 , values after rehabilitation vs values before rehabilitation. ${ }^{*} p<0.05$, group $B$ vs group $A$. apy $(\mathrm{p}<0.05)$. On admission, Group A patients had external rotation amplitudes of $14.20 \pm 4.98$ degrees, while on discharge the value was $25 \pm$ 6.46 degrees (Figure 2), which meant the $80 \%$ improvement. In Group B patients, the obtained effect was nearly half of this $-40.44 \%$.

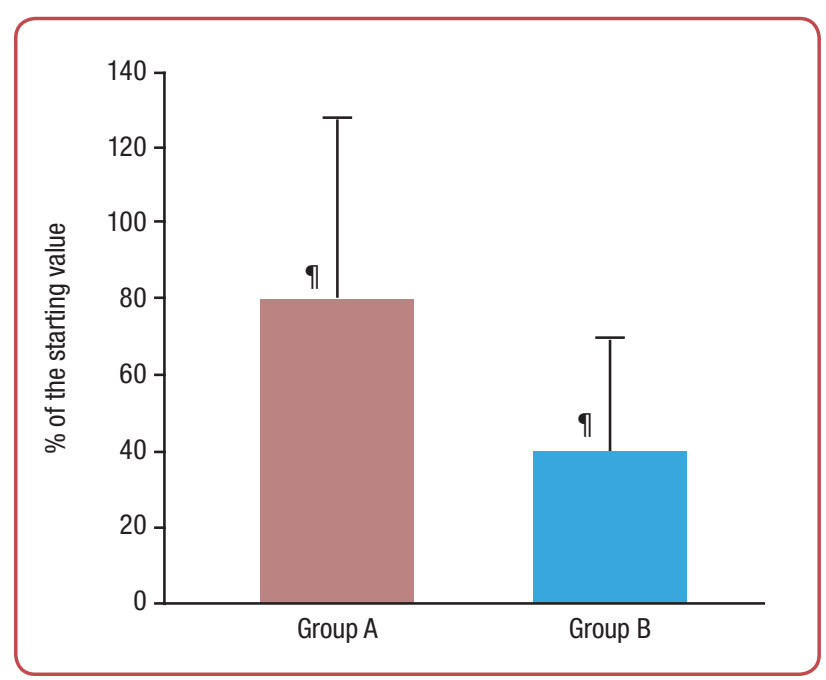

Figure 2: Increase in the amplitudes of the operated hip external rotation (\% of values before rehabilitation) in patients with hip replacement after in-patient rehabilitation. Group $A$ - rehabilitation programme plus hydrokinesitherapy, Group $B$ - rehabilitation programe without hydrokinesitherapy. $9 p<0.05$, values after rehabilitation vs values before rehabilitation.

${ }^{*} p<0.05$, group $B$ vs group $A$.

Although in both groups of patients significant improvements in amplitudes of other permitted movements in the operated hip were found on discharge after the in-patient rehabilitation, compared to the admission values (flexion with the flexed knee, extension and abduction), there were no significant differences between the Groups $A$ and $\mathrm{B}$.

\section{Femoral circumference}

Decreased the femoral circumference as a direct index of hypotrophy of the femoral muscles is one of the important parameters of the functional status of patients after total hip replacement. Although the increase of the femoral circumference $10 \mathrm{~cm}$ above the upper border of the patella was found in both groups of patients, it was significant $(\mathrm{p}<0.05)$ only in Group A patients $(51.92 \pm 3.23 \mathrm{~cm}$ on admission, $52.54 \pm 3.15 \mathrm{~cm}$ on discharge). The corresponding values in Group B patients were $51.94 \pm 3.27 \mathrm{~cm}$ and $52.36 \pm 3.36 \mathrm{~cm}$. Percentual changes in Groups A and B were $1.21 \%$ and 0.81 $\%$, respectively and this difference was significant (Figure 3). 


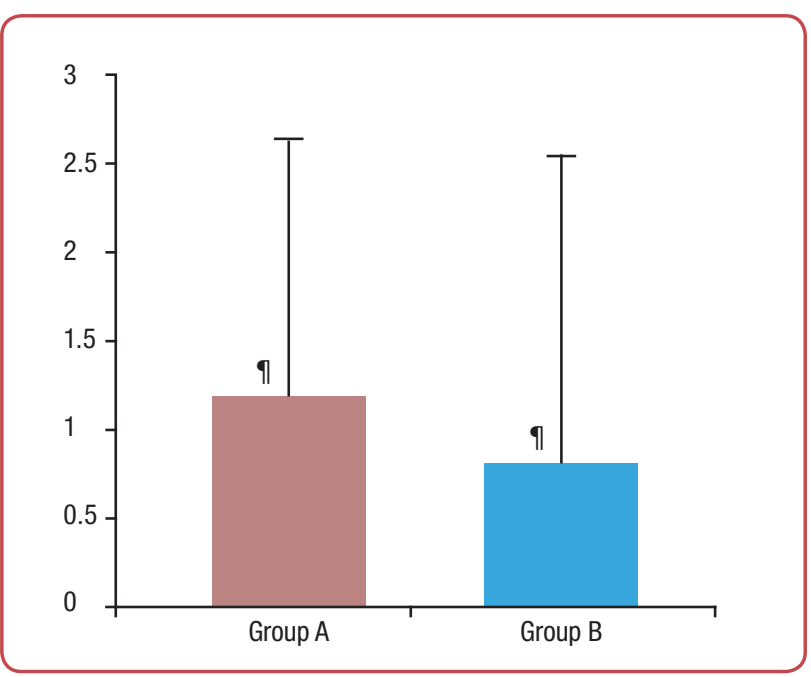

Figure 3: Increase in the vthigh circumference $10 \mathrm{~cm}$ above top border of the patella in patients with hip replacement after in-patient rehabilitation. Group A - rehabilitation programme plus hydrokinesitherapy, Group $B$ - rehabilitation programme without hydrokinesitherapy. $9 p<0.05$, values after rehabilitation vs values before rehabilitation. ${ }^{\star} p<0.05$, group $B$ vs group $A$.

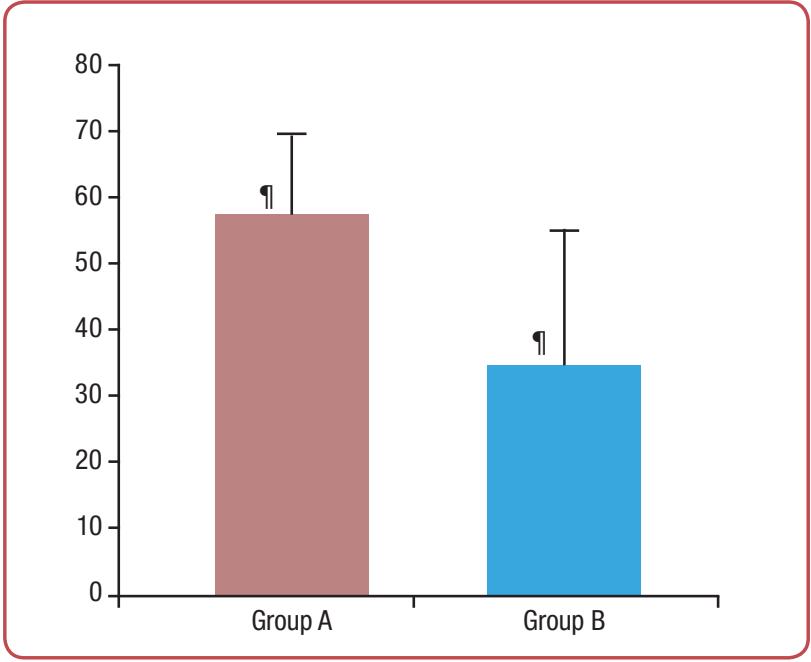

Figure 4: Decrease in the WOMAC index value in patients with hip replacement after in-patient rehabilitation. Group $A$ - rehabilitation programme plus hydrokinesitherapy, Group $B$ - rehabilitation programme without hydrokinesitherapy. $9 p<0.05$, values after rehabilitation vs values before rehabilitation. ${ }^{*} p<$ 0.05 , group $B$ vs group $A$.

\section{WOMAC index}

WOMAC index, as an index of the quality of life in patients after total hip replacement, was also significantly improved on discharge after the in-patient rehabilitation in Group A patients $(p<0.05)$. Mean value of this index on admission was 23.91 \pm 6.12 , while it was $10.66 \pm 4.78$ on discharge (Figure 4), which represents a $55.54 \%$ improvement. The same improvement of WOMAC index in Group B patients was 33.63\% and this difference was significant, in favour of the Group A.

\section{Discussion}

There is no universal rehabilitation protocol in the available literature that could serve as a "gold standard" for patients after total hip replacement. ${ }^{7-9}$ Various authors focus on the effects of different modalities of physical therapy and various kinesitherapy techniques. ${ }^{10-16}$

Results of the current research speak in favour of the benefits of the hydrokinesitherapy in rehabilitation of patients after total hip replacement. Analysis of the improvement in the amplitudes of movement in the operated hip, measuring the improvement of the muscular strength of the dynamic stabilisers of the hip and by testing the quality of life of these patients, it was shown that the biggest improvements were found in patients that underwent hydrokinesitherapy as a part of the complex in-patient rehabilitation treatment. At the same time, gender and BMI had no effect on the outcome of the in-patient rehabilitation in patients after total hip replacement. Although the Group A patients were significantly older, the outcome of their rehabilitation was better, which favours addition of hydrokinesitherapy to the standard in-patient rehabilitation protocol. There are several published clinical trials that support such a notion.

A group of Polish authors showed a significant improvement of the functional status in 96 patients undergoing hydrokinesitherapy after the implantation of the total hip replacement. ${ }^{17}$ Foley et al reported positive effects of hydrokinesitherapy on degenerative changes in the hip joint. ${ }^{18}$ They found that 105 patients undergoing hydrokinesitherapy had better results than the patients without such a treatment. The group of patients that had their exercises in the pool had a significantly improved muscular strength of hip stabilisers, combined with the increased amplitudes of the movements in the operated hip, which is confirmed by the present study, too. Belz et al estimated the positive effects of hydrokinesitherapy on the reeducation of the walking pattern in patients with artificial hip joint. Results of the research undertaken in 249 patients after a 20 -week-long hydrokinesitherapy (twice a week) clearly show that this type of therapy was efficient in the treatment of the degenerative diseases of the hip joint. Patients that exercised in water had significantly better results in comparison with the control group. ${ }^{19}$ Lin et al also reported on the beneficial therapeutic effect in 106 patients undergoing hydrokinesitherapy. 
Patients that had their exercises in water had significant improvements in the pain scores according to the VAS (Visual Analog Scale for pain) and in the amplitudes of movements in the operated hip. ${ }^{20}$ Siu et al reported better results in patients who exercised in water. ${ }^{21}$

In contrast to these results, Suomi et al did not support use of hydrokinesitherapy and found no significant changes in the muscular strength of hip stabilisers and hip joint mobility. ${ }^{22}$ These results were probably obtained because this study was performed on a small sample and with short total duration of hydrokinesitherapy. In fact, out of a total of 30 patients that were divided in three

\section{Conclusion}

Hydrokinesitherapy as part of the complex in-patient rehabilitation has a positive effect on increase of operated hip mobility, muscular strength and quality of life in patients after total hip replacement.

\section{References}

1. Singh JA. Epidemiology of knee and hip Arthroplasty: A systematic review. The Open Orthop J 2011;5(1):80-5.

2. Medical Advisory Secretariat. Physiotherapy rehabilitation after total knee or hip replacement: an evidence-based analysis. Ont Health Technol Assess Ser.2005;5(8):1-91.

3. Kim YS, Park J, Shim JK. Effects of aquatic backward locomotion exercise and progressive resistance exercise on lumbar extension strength in patients who have undergone lumbar diskectomy. Arch Phys Med Rehabil 2010;91(2):208-14.

4. Genet F, Gouin F, Coudeyre E, Revel M, Rannou F. The benefits of ambulatory physiotherapy after total hip replacement. Clinical practice recommendations. Ann Readapt Med Phys 2007;50:776-82.

5. Di Monaco M, Vallero F, Taperro R, Cavanna A. Rehabilitation after total hip arthroplasty: a systematic review of controlled trials on physical exercise programs. . Eur J Phys Rehabil Med 2009;45(3):303-17.

6. Di Monaco M. Rehabilitation after total hip and knee arthroplasty: where are we now? Work inprogress to built up evidence-based protocols. Eur J Phys Rehabil Med 2013;49(6):875-86.

7. Di Monaco M, Castiglioni C. Which type of exercise therapy is effective after hip arthroplasty? A systematic re- groups, only 10 patients underwent hydrokinesitherapy and its intensity was low - 2-3 times a week over 8 weeks.

Although there is a paucity of relevant data in the literature, based on the present study results and several available reports, the impression is that hydrokinesitherapy is an efficient method in rehabilitation of patients after implantation of the total hip endoprosthesis. Future similar studies should be organised on a larger sample of patients and they should be methodologically more precisely defined regarding the water temperature and composition, duration of the single treatment and the total number of the treatments received.

\section{Acknowledgements}

None.

\section{Conflict of interest}

None.

view of randomized controlled trials. Eur J Phys Rehabil Med 2013;49(6):893-907.

8. Levine B, Kaplanek B, Jaffe WL. Pilates training for use in rehabilitation after total hip and knee arthroplasty: a preliminary report. Clin Orthop Relat Res 2009;467(6):1468-75.

9. Aprile I, Rizzo RS, Romanini E, De Santis F, Marsan S, Rinaldi $\mathrm{G}$ et al. Group rehabilitation versus individual rehabilitation following knee and hip replacement: a pilot study with randomized, single- blind, cross-over design. Eur J Phys Rehabil Med 2011;47(4):551-59.

10. Minns Lowe CJ, Barker KL, Dewey ME, Sackley CM. Effectiveness of physiotherpy exercise following hip arthroplasty for osteoarthritis: a systematic review of clinical trials. BMC Musculoskelet Disord 2009 Aug 4;10:98. doi: 10.1186/1471-2474-10-98.

11. Barker AL, Talevski J, Morello J, Brand CA, Rahmann AE, Urquhart DM. Effectiveness of aquatic exercise for musculoskeletal conditions: a meta-analysis. Arch Phys Med Rehabil 2014;95(9):1776-86.

12. Kutzner I, Richter A, Gordt K, Dymke J, Damm P, Duda $G$ et al. Does aquatic exercise reduce hip and knee joint loading? In vivo load measurements with instrumented implants. Plos One 2017;12(2):102-16.

13. Liebs TR, Herzberg W, Rüther W, Haasters J, Russlies 
M, Hassenpflug J et al; Multicenter Arthroplasty Aftercare Project. Multicenter randomized controlled trial comparing early versus late aquatic therapy after total hip or knee arthroplasty. Arch Phys Med Rehabil 2012;93(2):192-9.

14. Tomaszewski W, Kotela I, Kawik L, Bednarenko M, Lorkowski J, Kotela A. Quality of live of patients in the evaluation of outcomes of short stem hip arthroplasty for hip osteoarthritis. Ortop Traumatol Rehabil 2013;15(5):439-57.

15. Li W, Ayers DC, Lewis CG, Bowen TR, Allison JJ, Franklin PD. Functional gain and pain relief after total joint replacement according to obesity status. J Bone Joint Surg Am 2017;99(14):1183-89.

16. Hoogeboom TJ, Stukstette MJ, de Bie RA, Cornelissen J, den Broeder AA, van den Ende CH. Non-pharmacological care for patients with generalizes osteoarthritis: Design of a randomized clinical trial. BMC Musculoskeletal Disord 2010;11:142-56.

17. Łyp M, Kaczor R, Cabak A, Tederko P, Włostowska E, Stanisławska I et al. A Water Rehabilitation Program in
Patients with Hip Osteoarthritis Before and After Total Hip Replacement. Med Sci Monit 2016;22:2635-42.

18. Foley NC, Bhogal SK, Teasell RW, Bureau Y, Speechley MR. Estimates of quality and reliability with the physiotherapy evidence-based database scale to assess the methodology of randomized controlled trials of pharmacological and nonpharmacological interventions. Phys Ther 2006;86(6):817-24.

19. Belz B, Topolski T, Kinne S, Patrick DL, Ramsey SD. Does adherence make difference? Results of community based aquatic exercise program. Nurse Res 2002;51(5):285-91.

20. Lin S, Davey RC, Cochrane T. Community rehabilitation for older adults with osteoarthritis of the lower limb: A controlled clinical trial. Clin Rehabil 2004;18(1):92-101.

21. Siu A, Chui D. Evaluation of community rehabilitation services for people wirh rheumatiod arthritis. Patient Educ Couns 2004;55(1):62-9.

22. Suomi R, Collier D. Effects of arthritis exercise programs on functional fitness and percieved acitivities of daily living measures in older adults with arthritis. Arch Phys Med Rehabil 2003;84(11):1589-94. 